\title{
Politicas educativas en \\ Latinoamérica: la vinculación entre los investigadores académicos y tomadores de decisiones en educación. Un análisis desde la teoria de los campos ${ }^{1}$
}

\author{
César Tello ${ }^{2}$ \\ Universidad Nacional de Tres de Febrero (UNTREF); ReLePe \\ Institución académica, Buenos Aires, Argentina \\ cesargeronimotello@yahoo.com.ar \\ Recibido: 1. de febrero de 2016 \\ Aceptado: 5 de julio de 2016 \\ Disponible en linea: 20 de diciembre de 2016
}

\begin{abstract}
Artículo de reflexión. Sin financiamiento. Este texto surge de la necesidad de explorar una línea analítica del ámbito de estudio de la relación entre productores de conocimiento y tomadores de decisiones en educación; particularmente, en lo que se refiere a los actores como pertenecientes a un campo, en términos de Bourdieu.

2 Profesor en Ciencias de la Educación, Universidad Nacional de La Plata(UNLP). Magíster en Políticas y Administración de la Educación, Universidad Nacional de Tres de Febrero (UNTREF). Doctor en Ciencias de la Educación, UNLP (Argentina). Investigador, UNTREF; y director de la Red Latinoamericana de Estudios Epistemológicos en Política Educativa (Buenos Aires, Argentina).
\end{abstract}




\title{
Politicas educativas en Latinoamérica: la vinculación entre \\ los investigadores académicos y tomadores de decisiones en educación. Un análisis desde la teoria de los campos
}

\section{Resumen}

La temática que aborda la relación entre producción de conocimientos y toma de decisiones en educación es un ámbito de estudios que se viene desarrollando desde la década de 1990. En la literatura especializada este tema se centra particularmente en las vinculaciones y relaciones que los actores deben establecer. Sin embargo, no se han planteado estudios acerca del rol social y político de los actores de esa relación. En el presente artículo se analiza esta dinámica desde la teoria de los campos, para comprender la complejidad de este ámbito de estudios que se hace cada vez más necesario en Latinoamérica.

Palabras clave: investigadores; tomadores de decisiones; politicas educativas

\section{Educational Policies in Latin America: The Link Between Academic Researchers and Decision Makers in Education. An Analysis from the Field Theory}

\begin{abstract}
The relationship between knowledge production and decision making in education is a field of study that has been developing since the 1990s. In specialized literature, this topic focuses particularly on the links and relationships that the various actors should build. However, no studies about the social and political role of these actors have been carried out. This paper analyzes those dynamics from Field Theory to understand the complexity of this area of study, increasingly necessary in Latin America.
\end{abstract}

Keywords: researchers; decision makers; education policies

\section{Politicas educacionais na América Latina: a relação entre pesquisadores acadêmicos e tomadores de decisão em educação. Uma análise desde a teoria de campo}

\section{Resumo}

A temática que aborda o relacionamento entre produção de conhecimento e tomada de decisão em educação é um âmbito de estudo que tem vindo a se desenvolver desde a década de 1990. Na literatura especializada este tema centra-se, particularmente, nas ligações e relações que os atores devem estabelecer. No entanto, não há estudos sobre o papel social e político dos atores nesse relacionamento. Neste artigo é analisada tal dinâmica desde a teoria dos campos, para compreender a complexidade de este âmbito de estúdios que resulta cada vez mais necessário na América Latina.

Palavras-chave: pesquisadores; tomadores de decisão; politicas educacionais 


\section{Presentación}

En Latinoamérica es alrededor de la década de 1990 que comienzan a desarrollarse, de un modo sistemático, ideas y reflexiones acerca de la relación entre la producción de conocimiento en educación y la toma de decisiones en la gestión educativa en el continente ${ }^{3}$. Sin duda que estas preocupaciones datan de la etapa de surgimiento de los propios Estados nacionales en Latinoamérica ${ }^{4}$, pero es solo en este último periodo que se ha comenzado a reseñar de un modo sistemático como ámbito de estudio y de desarrollo teórico.

Partimos de la premisa de que los estudios realizados, en general, se centran en los actores, esto es, los investigadores o productores de conocimiento y los tomadores de decisiones o gestores en educación, así como en la necesidad de acercamiento de unos y otros. Es aquí donde observamos algunas dificultades en la conceptualización que se establece en esta relación, ya que no hemos encontrado en la literatura especializada estudios en los que el análisis acerca de dicha relación se despliegue a partir del rol social que ocupan las partes, como tampoco que se asuman desde allí las particularidades y potencialidades de los actores que pertenecen a un campo social y político.

Por lo tanto, en este artículo desarrollamos este tópico tratando de partir de los actores sociales y de su rol en la red social y política, para analizarlos desde la perspectiva de 'campo' de Pierre Bourdieu. Así, revisamos y revisitamos algunas categorias analíticas y teóricas que despliegan propuestas de acercamiento entre ambos actores, que nos permiten desarrollar las ideas de este artículo, asumiendo que para Bourdieu un campo es un sistema de posiciones sociales y que cada

\footnotetext{
Véanse Brunner (1993); Tenti Fanfani (1994); Weiss (1994); Braslavsky y Cosse (1996); De Landsheere (1996); Cariola et al. (1997); Gibbons et al. (1997); Nazif y Rojas Figueroa (1997); Akkari y Perez (1998); Muñoz Izquierdo (2002); Corvalán (2003); Carrizo (2004); Cueto (2005); Ginsburg y Gorostiaga (2005); Botto (2008); Latapí (2008); Téllez (2008); Flores-Crespo (2009); Bracho (2010); Brunner (2010); Muñoz-Repiso (2010); Sancho (2010); Tello y Gorostiaga (2013).

4 Santiago Castro-Gómez (2000) explica que el surgimiento de las ciencias sociales no es un fenómeno posterior a los modos de organización política de los nacientes Estados nacionales en Latinoamérica, "sino constitutivo de los mismos" (p. 149). Se requería de "lineamientos científicos" sobre la realidad para fundamentar los modos de gobernar. Es decir: "Sin el concurso de las ciencias sociales, el Estado moderno no se hallaría en la capacidad de ejercer control sobre la vida de las personas, definir metas colectivas a largo y a corto plazo, ni de construir y asignar a los ciudadanos una "identidad" cultural" (p. 147).
} 
uno de ellos se define en relación a las otras posiciones. Un campo es (Moreno, 2003, p.16):

$[\ldots]$ un espacio específico en donde suceden una serie de interacciones $[\ldots]$ un sistema particular de relaciones objetivas que pueden ser de alianza o conflicto, de concurrencia o de cooperación entre posiciones diferentes, socialmente definidas e instituidas, independientes de la existencia física de los agentes que la ocupan.

Bourdieu (2004) define el campo como un espacio de lucha en donde se disputa el monopolio simbólico de lo que está en juego. Y aquí es necesario hacer una distinción metodológica entre producción de conocimiento y toma de decisiones en cuanto a la relación que se establece entre los campos.

Maggi (2003) explica que el problema de la relación entre unos (investigadores) y otros (gestores) no se da por la falta de comunicación, sino por las diferencias entre los marcos de referencia de los investigadores y de los agentes que toman decisiones en el ámbito de la gestión. Es decir, la vinculación se torna compleja si no comprendemos esos marcos de referencia a los que pertenecen los actores, esto es: los campos que se constituyen como "formas sociales, es decir, arbitrarias (relativas a un grupo particular) y socialmente determinadas" (Bourdieu, 2000, p. 66).

Por lo tanto, en este artículo presentamos inicialmente lo que consideramos una definición preliminar que sostiene el desarrollo del trabajo en términos de concepción y cosmovisión acerca de la producción académica del conocimiento. Analizamos luego las perspectivas y modelos analíticos en cuanto a los modos de nombrar la relación entre productores de conocimiento y tomadores de decisiones y modelos de relación. Así describimos el campo de los investigadores y el campo de los tomadores de decisiones, que nos permite, en el siguiente apartado, caracterizar la interfaz de vinculación desde los propios campos sociales a los que pertenecen unos y otros actores -y que hemos denominado espacio de la producción de conocimiento y la toma de decisiones (EPCYT)-, como un espacio que deberia desarrollarse y sostenerse 
a través de las políticas públicas en educación, para promover un nuevo formato que contribuya a la vinculación entre investigadores y tomadores de decisiones.

\section{Definición preliminar}

Es clave presentar una definición preliminar que le dará mayor claridad al desarrollo de este trabajo.

Asumimos en este artículo una concepción -entre otras posibles- de producción de conocimiento especializado en educación, como aquella que se da a partir de investigaciones de académicos, particularmente en ámbitos universitarios, y que poseen determinadas características: el recorte de un objeto de estudio, una metodología específica, información empírica y entrecruzamiento de ideas conceptuales con la información u observaciones realizadas por el investigador. Esta perspectiva es la comúnmente denominada actividad científica. Zorrilla (2010, p. 77) considera que:

[...] como actividad científica, la investigación educacional es un proceso pautado sobre el que existen consensos básicos que permiten cuidar y constatar su validez tanto interna como externa, así como la confiabilidad de sus hallazgos. Los dilemas y tensiones que están sobre la mesa de la discusión acerca de diversos asuntos teórico/epistemológicos, metodológicos y técnicos forman parte del proceso que permite el avance continuo en este campo científico.

Existen otras perspectivas sobre producción de conocimiento que podrian ubicarse en lo que denominamos conocimiento para la resolución de problemas.

La producción de conocimiento para la resolución de problemas, a diferencia del conocimiento académico, se caracteriza por surgir, principalmente, de una dificultad específica local, regional o de mayor magnitud geográfica y social; es un tipo de conocimiento producido en 
tiempos breves, con estilo de escritura tipo informe; en general, posee datos estadísticos y algunas categorías teóricas que le dan sustento, pero, a diferencia de la producción académica de conocimiento, las teorizaciones no son los ejes centrales, sino las recomendaciones para la acción y/o la toma de decisiones para la resolución de problemas.

En este sentido la OCDE-CERI (2004) distingue dos tipos de investigación y afirma que "la investigación básica está orientada a la producción de conocimientos en un área problemática determinada, de relevancia teórica o conceptual y tiende a ser disciplinar y más larga en el tiempo. La investigación de desarrollo, en cambio, tiene una orientación aplicada y contingente" (p. 266). En el mismo documento se denomina "investigación orgánica" al primer tipo de proceso de producción de conocimiento e "investigación instrumental" al segundo.

\section{Dos perspectivas y tres modelos analiticos}

Existen diversas perspectivas y modelos analíticos para establecer y nombrar las relaciones que se establecen entre productores de conocimiento y tomadores de decisiones. Sin embargo, en estas perspectivas y modelos se sitúa mayormente el énfasis sobre el actor y no sobre su pertenencia a un campo. En este apartado presentamos las reflexiones de Botto (2008) y Corvalán (2003), ya que consideramos que, entre la literatura especializada que se encuentra disponible, estas son las que poseen mayor grado de síntesis sobre el ámbito de estudio.

Botto (2008) plantea dos paradigmas: el Bridging Research \& Policy y el Embbeding Research \& Policy y argumenta que ambos se basan respectivamente en los enfoques racionalistas e incrementalistas de los estudios de las políticas públicas.

El primer paradigma, Bridging Research \& Policy (construyendo puentes entre la investigación y la política), se apoya en "una visión lineal y racional" (p. 27), en tanto los productores de conocimiento llevan adelante su tarea y los decisores se vinculan a ese conocimiento al 
utilizarlo de modo instrumental, llevándolo a la práctica de la gestión y la toma de decisiones. De este modo, la investigación social "provee" (p. 27) de conocimientos a los decisores. Y afirma la autora: "Estos roles no son intercambiables sino que los primeros fundan a los segundos" (p. 27). Este paradigma se caracteriza por la existencia de una necesidad de mejorar la articulación -vínculo y comunicación- entre productores de conocimiento y decisores.

El segundo paradigma, Embbeding Research \& Policy (encastrando la investigación y la política), según la autora sería más realista, ya que considera que existen múltiples modos en la vinculación entre estos actores "que se yuxtaponen y se autorregulan a través de un proceso de ajuste mutuo" (p. 28). Este paradigma critica la visión anterior, y complejiza la relación, entendiendo que aquí entrarian en juego diversos actores de un proceso de gestión en la toma de decisiones. Al no quedar reducida solo a las redes académicas existe mayor posibilidad de intercambio entre visiones y saberes consolidando democracias pluralistas y abiertas.

Por su parte, Corvalán (2003) utiliza una categoría que no es muy empleada al momento de pensar y analizar la relación entre productores de conocimientos y tomadores de decisiones, que creemos puede ser interesante para este trabajo: la "cadena de relaciones entre actores que son simultáneamente productores y usuarios de la información y conocimiento" (p. 17). En su argumentación, Corvalán afirma que estos actores "han estado por mucho tiempo desvinculados entre sî". Es decir, su propuesta gira en torno a un vínculo eslabonado que conformaria esta "cadena de relaciones", y explica (p. 24):

Cada eslabón de esta cadena forma parte importante del circuito de las decisiones centradas en los aprendizajes. Son los docentes, los directores de escuela, los estadísticos, los evaluadores, los investigadores y los planificadores de políticas educativas los que conforman esa cadena y que deben estar ligados entre sí para generar un proceso integrado de producción, análisis y uso de información y conocimiento significativo, llevando a un efectivo aprendizaje de los estudiantes. 
De modo concatenado a las formas de nombrar están los modelos de relación entre unos y otros actores. Es nuestra intención reseñar, en este artículo, tres modelos que consideramos que son útiles para la ilustración, pero que nunca se podrán encontrar delimitados como tales. De todos modos, contribuyen a comprender los campos -en el sentido de Bourdieu- que cada uno de estos grupos conforma.

Así podemos establecer un primer modelo que denominamos la producción del conocimiento como "lustre" (que da brillo). En este modelo el conocimiento da fuerza y sustento a las decisiones ya tomadas. Luego de la decisión se busca algún tipo de producción de conocimiento que les permita a los tomadores de decisiones justificar su accionar. Esto es lo que Weiss (1991) denomina "uso político", en tanto "su uso es selectivo para respaldar una posición adoptada previamente en relación a una decisión de política o de práctica" (Ginsburg y Gorostiaga, 2005, p. 287). En este sentido, y siguiendo la teoria de las dos culturas, dentro de la cultura de los decisores "el conocimiento es parcial, tendencioso e incompleto, es puesto al servicio de los propios intereses, y es políticamente acomodado" (Weiss, 1991, p. 175). Los tomadores de decisiones experimentan con agrado los "hallazgos" de investigaciones que coinciden con las decisiones tomadas; no solo con su perspectiva, sino con aquellas que, como dijimos, permiten justificarlas. Sin embargo, los tomadores de decisiones en este modelo casi nunca creen que las investigaciones les abran nuevas perspectivas sobre un determinado problema.

Planteamos un segundo modelo al que denominamos la producción de conocimiento como "apaga foco de incendio". Los resultados de investigación y el conocimiento son buscados cuando emerge un problema en la gestión. Un "foco de incendio". La investigación de tipo instrumental es clave en este modelo y se pudo haber realizado con anterioridad o realizarse rápidamente para resolver un problema que la gestión considera urgente. En este tipo de situaciones, los gestores, en general, reciben mucha presión social para que esa situación sea resuelta. Estas son investigaciones a demanda, para diagnosticar, resolver o evaluar situaciones concretas. Los investigadores y los 
tomadores de decisiones dialogan bajo los criterios de la conflictividad de las arenas politicas.

En este sentido, Moreles Vásquez (2009) afirma que "los tomadores de decisiones privilegian trabajos que permitan reaccionar ante situaciones urgentes, arreglos coyunturales, decisiones rápidas y no estudios interminables o que solo promuevan la incertidumbre" (p. 12).

Finalmente, el tercer modelo: la producción de conocimiento en un "doble rol". El modelo interactivo de Weiss (2009) se da de un modo particular en América Latina, pues, según la autora, los cursos de acción se reflexionan y deciden de manera conjunta; aquello que Reimers y MacGuin (1997) denominan "diálogos informados", García Godoy (2014) "modos alternados" o Ginsburg y Gorostiaga (2005) "membresía superpuesta". Es muy común encontrar dichas situaciones en algunos países de América Latina; generalmente se presentan cuando un investigador ocupa un cargo de gestión en la toma de decisiones y se vincula con los otros actores desde su doble rol. Según Moreles Vásquez (2009), "la incorporación de científicos a las esferas decisorias no resuelve los problemas de comunicación entre los grupos" (p. 3). En este modelo, según el autor, se termina por cooptar a los investigadores como otra manera de utilizar politicamente el conocimiento científico.

Tanto en las perspectivas como en los modelos analíticos se siguen avizorando buenas intenciones, y se analizan las instituciones sin tomar las caracteristicas del campo. Creemos que las disponibilidades a tender puentes, a conformar cadenas de relaciones y al encastramiento deben estar presentes. Pero nadie sabe muy bien en qué formato. $\mathrm{O}$, mejor dicho, cada uno se quiere vincular con el formato propio de su campo y es alli, como dijimos, donde radica la gran dificultad: en tratar de encastrar piezas de un puzzle que nunca podrán unirse si no se asume el campo al que pertenecen, porque se hablan lenguajes distintos y se poseen intereses diversos. Nuestra propuesta consiste entonces en comenzar a generar y pensar un nuevo formato: el de la interfaz entre productores de conocimiento y tomadores de decisiones, considerando las características de los propios campos. 


\section{El campo de los investigadores y el campo de los tomadores de decisiones}

Definiremos qué entendemos por campo para cada uno de los actores, y tomando la producción de Pierre Bourdieu a este respecto, debemos considerar que las categorías generales son bien conocidas por los lectores.

Sin duda que no podemos desarrollar la noción de campo de Pierre Bourdieu sin la categoria de habitus, dado que la estructura en la que el habitus opera es la que el sociólogo francés ha denominado campo. Entre habitus y campo existe una relación dialéctica en el sentido de que el habitus, formado en un campo específico, es capaz de instituir acciones en las que cobra sentido (Bourdieu, 2002), mientras que en otro campo esas acciones podrian ser vistas como "no comunes" o "conductas extrañas".

De este modo, y siguiendo el análisis de Meischner (2007), podemos argüir que los diversos modos y formas de la práctica tienen lugar entre la interacción del habitus de un sujeto que trae consigo las condiciones/limitaciones/inherencias propias del campo en el cual realiza sus acciones sociales junto a los otros actores del campo. Esto es: "microcosmos relativamente autónomos" (Bourdieu y Wacquant, 1996, p. 127).

Así, el campo de los tomadores de decisiones en educación se conforma con determinadas características homologables al campo político de Bourdieu. En este sentido, Meischner (2007, p. 19) afirma:

El volumen y la estructura del capital de que dispone un politico, tal como las reglas del juego específico del campo político, determinan el papel que se asigna al político y que limita su espacio de acción y de creación. [...] Pero las reglas del juego dentro del campo no limitan solamente, sino que facilitan también de prever las posiciones que tomarán los otros políticos y también que el grupo mismo sea previsible para los demás. Todos los participantes del juego están relacionados entre ellos por un acuerdo fundamental al juego, a las 
reglas del juego, a lo que está en juego y por una solidaridad fundamental. Esta unión es más fuerte que todos los acuerdos abiertos o secretos y evita los cuestionamientos sobre las propias reglas del juego siendo aún válidos.

Entretanto el campo de los investigadores es homologable al campo científico y académico, considerando en primer lugar que, tal y como afirma Bourdieu (2002, p. 47):

La idea de una ciencia neutra es una ficción, y es una ficción interesada, que permite considerar científica una forma neutralizada y eufemística (y por lo tanto particularmente eficaz simbólicamente porque es particularmente desconocible) de la representación dominante del mundo social.

Bourdieu no solo no acepta la supuesta neutralidad de la ciencia, sino que va mucho más allá cuando afirma: "la ciencia social toma necesariamente partido en la lucha política" (2002, p. 48). Con esto decimos que no aceptamos la idea de "los investigadores en su torre de marfil", por el contrario, creemos que un investigador en educación debe participar del conocimiento sensible (Tello, 2006), es decir, del compromiso social a través de sus producciones. Aun así, asumiendo la presencia de esta característica por parte de los investigadores no se obtiene per se la vinculación con los tomadores de decisiones, dado que es necesario considerar, como hemos mencionado, los lenguajes, lógicas y "modos de hacer" de cada campo.

Ahora bien, siguiendo las argumentaciones de Bourdieu (2002) y su categoría sobre el campo, podemos observar que su preocupación radicó en la posibilidad de protagonismo del campo de los investigadores, precisamente para no perder su identidad como tales, dado que cuanto mayor sea el grado de autonomía y legitimación específica en ciencias sociales, mayor será su autonomía frente a la incidencia que pudieran tener otros campos, en nuestro caso el político o el de la gestión. 
Respecto a las agendas y las problemáticas planteadas anteriormente, debemos considerar que Bourdieu (2002) realizaba una advertencia: "el intelectual es un personaje bidimensional: solo existe y subsiste como tal si, por una parte, existe y subsiste un mundo intelectual autónomo, es decir, independiente de los poderes religiosos, políticos y económicos" (p. 187). Así, Bourdieu (2002) afirma que esta autonomía se encuentra amenazada, dado que existe un "imperio de la economía" sobre investigación, vinculada a las nuevas formas de gobernar en el marco de la globalización.

La investigación es acción sociopolítica porque genera nuevas miradas y ángulos sobre la realidad, pero no es gestión sociopolítica. Una relación constructiva supone reconocer, respetar y validar las diferentes identidades, roles, objetivos, reglas y restricciones de cada una de las partes (Zorrilla, 2010).

Moreles Vásquez (2009) afirma que "la política también influye en la investigación, y sus efectos más representativos son la planificación de nuevos temas de investigación, en lo que concierne al ámbito científico, y la modificación del discurso público y de iniciativas, en lo que respecta al político" (p. 13). No obstante, en su estudio el autor observa que "los investigadores se guían más por las necesidades inmediatas de su entorno académico que por las de los usuarios potenciales del conocimiento, y que por lo tanto el origen de problemas y agendas de investigación es principalmente disciplinario o teórico" (p. 15).

Cada uno de los actores que mencionamos pertenece a un campo, donde hay luchas de poder hacia el interior del mismo, en el que hay representaciones sociales acerca de quiénes deben ser y sobre cómo los ven el resto de los actores sociales. En la mayoria de los estudios sobre la temática no se indica esto con claridad, por lo que se pueden argüir propósitos ingenuos de vinculación. En síntesis, tenemos dos campos atravesados por la dimensión política: 1) el campo de los tomadores de decisiones atravesado con fuerte prescripción del eje político partidario; 2) el campo de los investigadores atravesado por agendas de investigaciones, a veces impuestas por el propio campo; 
otras, por sus propias preocupaciones alejadas de la realidad del campo de los tomadores de decisiones.

Definimos el campo de los investigadores y de los tomadores en tanto construcciones sociales y culturales en movimiento, como afirma Suasnábar (2009): con límites de porosidad entre los campos, advertencia que no desplegaba la tesis de las dos culturas, atendiendo a las críticas que esgrimieron Ginsburg y Gorostiaga (2005), quienes afirman (p. 289):

Aunque los temas discutidos por la tesis de las dos culturas son instructivos, este modelo explicativo, como cualquier otro, puede exagerar o simplificar en demasía en su intento por iluminar ciertos aspectos importantes de la experiencia humana. Nos detendremos en tres elementos estereotípicos de la descripción de las dos culturas: (1) presenta sólo un retrato dominante de cada cultura; (2) ignora la heterogeneidad de los miembros de cada grupo; y (3) exagera la pertenencia de los individuos a uno solo de los dos grupos culturales.

Dado que los campos constituyen espacios de disputa de las fuerzas sociales, ya sea por conservar o transformar los capitales, tanto el campo político como el de la investigación son dinámicos y se entrecruzan: "El campo es escenario de relaciones de fuerza y de luchas encaminadas a transformarlas y, por consiguiente, el sitio de un cambio permanente" (Bourdieu, 2002, p. 69).

Biddle y Anderson (1991) afirman que "la frustración acerca del nivel de comunicación entre teóricos/investigadores y decisores/profesionales se basa, en parte, en malentendidos acerca de la forma en que su conocimiento puede afectar una institución como la educación" (citado en Ginsburg y Gorostiaga, 2005, p. 290).

Nos preguntamos, entonces, ¿ambos actores deben cambiar para que se produzcan vinculaciones? Si se les piden cambios a los actores sociales de ambos campos, ¿¿seguirán cumpliendo su rol? Y, por lo tanto, ¿tendrán estos actores potencialidad para la transformación de la realidad? 
El debate sobre esta cuestión se torna dicotómico y bidireccional, dado que en ocasiones solo se analiza la relación desde uno de los campos. Desde la perspectiva de los investigadores:

1. Están quienes consideran que el espacio que deben ocupar es el de la investigación, y que acercarse a la gestión puede generar cierta "contaminación" en las investigaciones como en sus agendas, dado que la mayoria de los investigadores en educación asumen que una situación es estar atentos a la realidad y otra muy distinta "atentos a la gestión".

2. También se puede encontrar a quienes consideran importante que los investigadores estén cerca de la gestión y atiendan la demanda de la gestión, algunos con ciertos cuidados, otros a cualquier costo, incluso realizando "informes rápidos porque los necesita el ministro". Y aquí también se debe incluir la diversidad de temas que un funcionario/tomador de decisiones debe abarcar y la disposición de un investigador especialista para responder a ello.

3. En tercer lugar mencionamos a aquellos que consideran que hacen algún aporte importante para la gestión y que tratan de difundir sus resultados de investigación en estas esferas, convirtiéndose, en ocasiones, en investigadores mediáticos. Lo que no es negativo en sí mismo, aunque en Latinoamérica se observa que son buscados por los medios para temas de "educación" y que se les consulta por temas disímiles como educación superior, repitencia en la escuela primaria, cambios curriculares de programas, entre otros. Quizá aquí la pregunta no sea por qué los medios eligen a ese investigador, si no por qué el investigador responde a todos los temas.

Desde la perspectiva de los gestores:

1. Quienes "necesitan" del conocimiento producido por los investigadores para justificar sus decisiones y entonces toman investigaciones para sus decisiones o incorporan investigadores a sus equipos técnicos, con el fin de darles cierto cariz de "rigurosidad 
intelectual" a las decisiones como decisiones tecno-políticas "barnizadas" de cientificidad (Weiss, 2009).

2. Quienes consideran que el conocimiento producido es importante para la toma de decisiones, y les solicitan a los investigadores determinadas indagaciones o los incorporan a los equipos técnicos como "académicos" para la consulta.

3. Quienes consideran que existen investigaciones que pueden ser muy interesantes para resolver problemas de la gestión.

Esta es la situación. Por tanto, pensar en la vinculación entre unos y otros siempre resulta conflictivo, precisamente porque todos ocupan roles sociales diversos. Las representaciones que sobre ellos recaen, así como las que ellos tienen de sí mismos a través de la construcción del rol, nos obligan a pensar los modos de vinculación desde estos lugares: los de los productores de conocimiento y los de los tomadores de decisiones. Igualmente, estas nos conducen a pensar otros lugares, lugares no existentes aún. Aquí hay que señalar que lo conflictivo no se resolverá con la realización de una reunión entre tomadores de decisiones e investigadores, como tampoco con buenas intenciones para sentarse en la misma mesa, porque el proceso de vinculación e interacción va mucho más allá del "estar juntos" y del tender puentes como meros espacios de acercamiento y diálogo.

$\mathrm{El}$ puente es un lugar que conecta, que vincula, pero en el modo tradicional, ya que un puente une dos partes geográficas (actores sociales) distintas, distantes. Nadie puede vivir en el puente. En ese sentido, el establecer puentes no conlleva abordar modos distintos de relación entre productores de conocimiento y tomadores de decisiones. Es necesario crear una interfaz, un espacio de intermediación, para que esto suceda. Pero no tiene nada que ver con un espacio geográfico, ni con reuniones, sino con algún modo de vinculación que les permita a ambos involucrarse en un mismo espacio social. 


\section{La Interfaz: espacio de la producción de conocimiento y la toma de decisiones (EPCYT)}

Tras haber planteado el lugar desde donde comprendemos a los actores clave de esta relación y cómo se produce conocimiento desde los investigadores académicos; las perspectivas y modelos analíticos en los modos de nombrar la relación entre unos actores y otros; y habiendo diferenciado el campo de pertenencia de los investigadores y tomadores de decisiones, caracterizaremos la interfaz que estamos proponiendo como modo de vinculación.

En primer lugar, debemos considerar que la interfaz no es consultoria, no es investigación a demanda; es proyecto de superación de la realidad que antecede a una investigación. En caso de que no haya investigación rigurosa y de calidad, sí es consultoría, lo que bajo ningún aspecto es negativo, pero para nosotros no es vinculación entre productores de conocimiento y tomadores de decisiones, porque se eliminan componentes propios del campo; debemos tener en cuenta que, en la consultoría, la gestión solicita información a investigadores para un determinado problema. En este caso estariamos ante otro actor sobre el que no hemos indagado en este trabajo: el consultor.

El consultor es aquel especialista -investigador académico o noque contrata el gobierno u otro organismo a través de un tomador de decisiones para una determinada tarea, sea en forma personal o a través de un think tank u otro tipo de entidad.

Klobucký y Strapcová (2004) sostienen que las investigaciones que quieran tener impacto sobre los tomadores de decisiones deben sugerir recomendaciones, cuestiones prácticas y condiciones necesarias para el éxito de esa propuesta. Los autores centran la producción de los investigadores en el impacto; no cabe duda de que esta propuesta mercadológica de impacto no llega a generar un espacio de conjunción y vinculación, y que es más riesgosa que los modelos de producción de conocimiento que hemos desarrollado en este trabajo, en particular porque aquí se establece la necesidad de "salir a ofrecer" el producto, desdibujando uno y otro rol nuevamente. 
Nosotros proponemos otro espacio. Esto no implica que deba incluirse a otros actores (bróker de información, tecno-político, etc.), como tampoco la transformación de los actores: analistas simbólicos, expertos, etc. Se trata, por el contrario, de un espacio donde ambos actores den y generen un espacio social, un nuevo campo: una interfaz. Es en la interfaz donde conviven, donde debaten y donde desde sus propios aportes se genera un proyecto de superación de la realidad. Debe considerarse que la vinculación entre productores de conocimientos y tomadores de decisiones tiene su eje de debate en la transformación de la realidad social. Sin duda que los tomadores de decisiones pueden contribuir al campo académico con sus reflexiones, pero hemos dejado claro que el conocimiento no puede quedar atado a los vaivenes de la arena política de la gestión, aunque no quede excluida de ella.

En este sentido, se debe generar una política educativa cuyo objeto sea la creación de interfaces. En la actualidad, en contraste, se observan politicas educativas que se vienen aplicando en varios países de Latinoamérica destinadas a financiar "la investigación aplicada". Ahora bien, considerando las argumentaciones realizadas en este trabajo al respecto, ¿cuáles serian las características de la investigación aplicada? ¿investigación para la toma de decisiones: consultorias, recetas-recomendaciones? Estas siguen siendo políticas "estilo puente" y dicotómicas.

La investigación no puede valorizar qué cuestión resulta mejor para la toma de decisiones. Por eso sería un error conceptual situarse en una u otra posición para explicar la relación entre los actores; el espacio de la producción de conocimiento y la toma de decisiones: EPCYT, es decir, la interfaz de la convergencia de los actores que no responden ni al modo 1 ni al 2, en términos de Gibbons et al. (1997) ${ }^{5}$, teniendo en cuenta que en el EPCYT la convergencia se produce por los aportes, pero no a modo de identificación de actores sino como una

5 Según estos autores, existen dos modos: el "modo 1" que se definiría como el modelo tradicional de producción del conocimiento, en el cual lo estrictamente técnico y epistemológico disciplinar guía el proceso de investigación. En contraposición, el "modo 2" es orientado por las necesidades del contexto de aplicación e incluye redes de trabajo amplias, temporarias y heterogéneas, que colaboran para definir y resolver problemas en un contexto local y específico. 
construcción conjunta con características particulares de cada uno de los actores del campo, como un espacio de debate y reflexión.

Puede decirse que el EPCYT es diferente al "doble rol", a los "diálogos alternados" y a los "modos informados". La diferencia radica en que, en este espacio, el eje de la construcción no reside en los resultados de investigación del investigador, sino en el tema que los convoca y a partir del cual se genera el EPCYT; el eje es la construcción de un Proyecto de Superación de la Realidad.

Sería un grave error que la inversión pública se destine a centros de investigación tipo think tank o consultorias, porque al contratarlos los hacen presos de sus agendas. En cambio, invertir financiamiento público en espacios de EPCYT permite que los actores sociales puedan seguir perteneciendo a sus campos.

Por su parte, Cariola et al. (1997) esgrimen la necesidad de un nuevo agente, o lo que nosotros denominamos agente metamorfoseado, enfatizando en "la intermediación entre el conocimiento y la acción" en el ámbito educativo (p. 25). Según este planteo, el analista de políticas o "broker de información" es un profesional "con habilidades específicas para aplicar el método de la investigación en ciencias sociales a la investigación de problemas desde la perspectiva del tomador de decisiones" (p. 26). Esto implica que la responsabilidad recae sobre un sujeto, un "puedelotodo", sin que se comprendan la pertenencia ni las diferencias de los campos de los que puede hacer parte el "bróker de información", lo que lo encierra en un espacio que no es el suyo; en general, como hemos mencionado anteriormente, promoviendo el "doble rol".

También es necesario señalar que en la búsqueda bien intencionada de investigadores de este ámbito de estudio se producen propuestas que terminan por desdibujar el proceso. Weiss (1994) plantea tres modos de producción de conocimiento que permitirian mayores posibilidades con los tomadores de decisiones, pero que para nosotros poseen ciertos riesgos, principalmente por la no-presencia de un 
espacio de vinculación, de una interfaz que consideramos que es clave para que puedan tener potencialidad ambos actores. Ellos son:

1. Investigación en líneas sostenidas, desarrollada con autonomía en instituciones universitarias y centros de investigación y desarrollo, financiados sin agendas temático-metodológicas predefinidas, pero a partir de programas integrales.

2. Estudios específicos, como diagnósticos, evaluaciones o prospectivas, para generar, a corto plazo, la información requerida por los gobiernos u organismos. Se recomienda contratarlos externamente.

3. Prototipo en diseño de sistemas de información y análisis, de sistemas sectoriales, de gestión y organización institucional, de currículo, de programas de formación y de materiales didácticos, con una mayor participación de los grupos de investigación.

Aunque la intención de Weiss, como afirman Krocht y Suásnabar (2002) fue principalmente "rescatar y defender la producción de conocimiento desinteresada" (p. 12), creemos que con las propuestas 2 y 3 no hace más que reafirmar el "modelo de consultoría".

En definitiva, ninguna de estas propuestas establece una interfaz de compromiso mutuo: el proyecto o programa de superación de la realidad o de la práctica. Es decir, ese espacio donde productores de conocimientos y tomadores de decisiones piensan y reflexionan sobre la realidad a partir de ideas, de nuevas concepciones teóricas: la interfaz de la vinculación, que no se lleva a cabo exclusivamente con la lógica de un campo ni del otro.

\section{Conclusiones}

Finalmente cabe señalar que la propuesta de la interfaz es una advertencia ante los embates del neoliberalismo, ya que debemos tener en cuenta que este ámbito de debate surge hacia finales de la década 
de 1980, cuando comienzan a emerger los think tanks, generalmente financiados por agencias y fundaciones internacionales, así como por empresas locales. Estos se erigen en América Latina como espacios en los que los investigadores adoptan los nuevos roles de consultores y brókers del conocimiento. Siguiendo la línea de análisis de Beltrán (2005), desde el punto de vista empresarial el interés está puesto en apostar a que los intelectuales generen información, interpretaciones y dispositivos que les permitan influir en los politicos para realizar cambios en las políticas estatales que los beneficien económicamente. En cambio, los intelectuales expertos persiguen intereses propios de posición política, académica o económica (véase Simón, 2006).

En los últimos años, los científicos sociales más destacados se transformaron en consultores de los centros establecidos en los países de Latinoamérica o en centros regionales (De Sierra et al., 2006). Así, la sociología latinoamericana ha experimentado la transformación de algunos antiguos centros de investigación "en empresas de consultoría, fenómeno que se observa en casi todos los países en la región" (Borón, 2006, p. 5).

La producción de conocimiento de los think tanks se caracteriza, como lo señala Brunner (1993), "por tener la capacidad para aprovechar efectivamente los conocimientos disponibles" (p. 11) y no tanto por desarrollar investigaciones rigurosas y tradicionales. Según el autor: se caracterizan por producir conocimiento "útil".

Tenti Fanfani (1994) denuncia el conocimiento "útil" que propone Brunner y plantea que esa perspectiva es apropiada para ganar en las transacciones y negociaciones donde se diseñan las decisiones. Además, explica que, de esta forma, el viejo criterio de verdad que orientaba a la ciencia clásica es reemplazado por el criterio de utilidad. En una línea similar, Weiss (1994) critica la propuesta de Brunner y argumenta que "la teoría demasiado comprometida con la acción directa, los gobiernos y los poderes económicos pierde su dimensión crítica y se convierte fácilmente en esclava de malas prácticas" (p. 14). 
Nosotros no tenemos dudas de que el conocimiento debe estar comprometido con la realidad social. Sin embargo, después del análisis que realizamos en este trabajo, podriamos afirmar que sería ingenuo pensar que el conocimiento per se se traslada hasta las esferas de los tomadores de decisiones e ilumina a los gestores en la arena politica. Por tales razones, asumimos, desde la perspectiva de los campos, que la producción de conocimiento y la toma de decisiones se da a través de actores y que esos actores requieren de espacio para intercambiar, dialogar, proyectar. Pero también consideramos que se debe generar ese espacio que le permita a cada actor ser lo que es, en el que ni uno ni otro deba renunciar a sí mismo, ni ser absorbido por las lógicas de las políticas neoliberales.

\section{Referencias}

Akkari, A. y Perez, S. (1998). Educational Research in Latin America: Review and Perspectives. Education Policy Analysis Archives, 6(7), 1-10.

Beltrán, G. (2005). Los intelectuales liberales. Poder tradicional y poder pragmático en la Argentina reciente. Buenos Aires: Libros del Rojas - UBA.

Biddle, B. y Anderson, D. (1991). Social Research and Educational Change. En D. Anderson y B. Biddle (Eds.), Knowledge for Policy: Improving Education through Research (pp. 1-20). London: Falmer.

Borón, A. A. (2006). Las ciencias sociales en la era neoliberal: entre la academia y el pensamiento crítico. Tareas, 122, 45-73.

Botto, M. (2008). Saber y politica en América Latina. El uso del conocimiento en las negociaciones comerciales internacionales. Buenos Aires: Prometeo.

Bourdieu, P. (2000). Intelectuales, politica y poder. Buenos Aires: Eudeba.

Bourdieu, P. (2002). Cosas dichas. Barcelona: Gedisa. 
Bourdieu, P. (2004). Los usos sociales de la ciencia. Buenos Aires: Nueva Visión.

Bourdieu, P. y Wacquant, L. (1996). Una invitación a la sociología reflexiva. Buenos Aires: Siglo XXI Editores.

Bracho, T. (2010). Politicas basadas en evidencia: la politica pública como acción informada y objeto de investigación. En G. Cejudo (Comp.), Problemas, decisiones y soluciones: enfoques de política pública (pp. 291-319). México: Fondo de Cultura Económica.

Braslavsky, C. y Cosse, G. (1996). Las actuales reformas educativas en América Latina: cuatro actores, tres lógicas y ocho tensiones. Santiago de Chile: PREAL.

Brunner, J. J. (1993, junio). Investigación social y decisiones politicas: el mercado del conocimiento. Ponencia presentada en el Seminario "La Investigación Educacional Latinoamericana", Consejo Latinoamericano de Ciencias Sociales, Punta Tralca, Chile.

Brunner, J. J. (2010). La investigación educacional en Chile: Retos por delante. Ponencia presentada en el Congreso Interdisciplinario de Investigación en Educación, Santiago de Chile.

Cariola, P., Schiefelbein, E., Swope, J. y Vargas, J. (1997). La vinculación entre la investigación y la toma de decisiones en educación: un nuevo enfoque. Santiago: CIDE.

Carrizo, L. (2004, agosto). Producción de conocimiento y políticas públicas. Desafios de la universidad para la gobernanza democrática. Revista Reencuentro, $40,1-15$.

Carrizo, L. (2004, diciembre). Producción de conocimiento y políticas públicas. Desafios de la universidad para la gobernanza democrática. Cuadernos del CLAEH, 89 [Montevideo].

Castro-Gómez, S. (Ed.). (2000). La reestructuración de las ciencias sociales en América Latina. Bogotá: Instituto PENSAR. 
Corvalán, A. M. (Coord.). (2003). La información y el conocimiento. Un desafio para lograr una gestión de la educación al servicio de los aprendizajes. Santiago de Chile: UNESCO.

Cueto, S. (Ed.). (2005). Uso e impacto de la información educativa en América Latina. Chile: PREAL.

De Landsheere, G. (1996). La investigación educativa en el mundo. México: Fondo de Cultura Económica.

De Sierra, G. et al. (2006). Las ciencias sociales en América Latina en una mirada comparativa. En H. Trindade (Org.), Las ciencias sociales en América Latina en perspectiva comparada (pp. 17-52). México: Siglo XXI.

Flores-Crespo, P. (2009) Investigación educativa y políticas públicas en México: una relación amorfa y elusiva. Sinéctica Revista Virtual de Educación. Recuperado de http://www.scielo.org.mx/scielo.php?script=sci_arttext\&pid=S1665109X2009000200005

García, B. (2014). Producción de conocimiento científico y toma de decisiones en el campo de la política pública. Modelos, tensiones y perspectivas. Revista Debate Público. Reflexión de Trabajo Social, 4(8).

Gibbons, M. et al. (1997). La nueva producción del conocimiento. Barcelona: Pomares Corredor.

Ginsburg, M. y Gorostiaga, J. (2005). Las relaciones entre los teóricos/ investigadores y los decisores/profesionales: repensando la tesis de las dos culturas y la posibilidad del diálogo en el sector educativo. Revista Española de Educación Comparada, 11, 285-314.

Klobucký, R. y Strapcová, K. (2004). Knowledge utilization in public policy: the case of Roma population research in Slovakia. International Social Science Journal, 56(179), 57-73. 
Krotsch, P. y Suasnábar, C. (2002). Los estudios sobre la Educación Superior: una reflexión en torno a la existencia y posibilidades de construcción de un campo. Revista Pensamiento Universitario, 10, 35-54.

Latapí, P. (2008). ¿Pueden los investigadores influir en la politica educativa? Revista Electrónica de Investigación Educativa, 10(1). Recuperado de http:// redie.uabc.mx/vol10no1/contenido-latapi2.html Consultado el 4/6/2009

Maggi, R. (2003). Usos e impactos de la investigación educativa. En E. Weiss (Coord.), El campo de la investigación. Colección la investigación educativa en México (1992- 2002) (pp. 193-236). México: COMIE.

Meischner, S. (2007). El campo político en la perspectiva teórica de Bourdieu. Iberóforum. Revista de Ciencias Sociales de la Universidad Iberoamericana, II(3), 1-22.

Moreles Vázquez, J. (2009). Uso de la investigación en la política. Una aproximación a su estudio. Archivos Analiticos de Políticas Educativas, 17(13). Recuperado de http://epaa.asu.edu/epaa

Moreno A. (2003). Introducción elemental a Pierre Bourdieu. Bogotá: Álvaro Moreno.

Muñoz Izquierdo, C. (2002, julio). Investigación educativa y políticas públicas. Texto preparado para el panel sobre experiencias en el uso del conocimiento generado por los investigadores educativos, llevado a cabo en el Taller sobre el mismo tema, Ouro Preto, MG., Brasil.

Muñoz-Repiso, M. (2010). Investigación, política y práctica educativas. Revista Iberoamericana sobre Calidad, Eficacia y Cambio en Educación, 8(2), 201-216.

Nazif, M. y Rojas Figueroa, A. (1997). La investigación educativa latinoamericana en los últimos diez años. Revista de Educación, 312, 21-42.

OCDE-CERI. (2004). Revisión nacional de investigación y desarrollo educativos. Reporte de los examinadores sobre México. OCDE. 
Reimers, F. y McGinn, N. (1997). Informed dialogue: Using research to shape education policy around the world. Westport, CT: Praeger.

Sancho, J. (2010). Del sentido de la investigación educativa y la dificultad de que se considere para guiar las politicas y las prácticas. Revista Iberoamericana sobre Calidad, Eficacia y Cambio en Educación, 8(2), 34-46.

Simón, J. (2006). Entre la ciencia y la politica: los think tanks financiados por el empresariado y la producción de conocimiento en educación en Argentina (Tesis de maestria). FLACSO, Buenos Aires.

Suasnabar, C. (2009, abril). ¿Pedagogos criticos, expertos en educación, tecnopolíticos, o qué? A propósito de la relación entre intelectuales de la educación y politica en los últimos 50 años. Ponencia presentada en el Seminario "Elites intelectuales y formación del Estado", Buenos Aires, Argentina.

Téllez T., F. (2008). Redes de Producción y Diseminación de Información sobre Educación en América Latina y el Caribe: su utilidad para Tomadores de Decisión. Archivos Analiticos de Politicas Educativas, 16(14).

Tello, C. (2006). El conocimiento como experiencia sensible: la formación universitaria. Desafios latinoamericanos [Biblioteca Digital de UNESCO-IESALC, Instituto Internacional para la Educación Superior en América Latina y el Caribe, Venezuela]. Boletín Digital IESALC, 115.

Tello, C. y Gorostiaga, J. (2013). Las relaciones entre investigadores y tomadores de decisiones: discusiones en torno al concepto de "analista simbólico" y el enfoque de las "políticas basadas en evidencia". En N.G. Gutiérrez Serrano (Comp.), Formación, politica e investigación. Espacios de producción de conocimiento en educación en México y el Cono Sur (pp. 101-134). México: UNAM - Plaza y Valdez.

Tenti Fanfani, E. (1994). Del Intelectual Orgánico al Analista Simbólico. Revista de Ciencias Sociales, 1, 19-29. 
Weiss, C. (1991). The Many Meanings of Research Utilization. En D. Anderson y B. Biddle (Eds.), Knowledge for Policy: Improving Education through Research (pp. 173-182). London: Falmer.

Weiss, E. (1994). ¿Cómo consolidar la investigación educativa? Revista Universidad Futura 6(16), 12-16.

Weiss, C. (2009). Prólogo. En F. Carden (Ed.), Del Conocimiento a La Política. Máximo aprovechamiento de la investigación para el desarrollo (pp. 19-23). Ottawa: Centro Internacional de Investigaciones para el Desarrollo.

Zorrilla, M. (2010). Investigación Educativa, Políticas Públicas y Práctica Docente. Triángulo de Geometría Desconocida. Revista Electrónica Iberoamericana sobre Calidad, Eficacia y Cambio en Educación, 8(2), 74-92.

\section{Cómo citar este artículo}

Tello, C. (2017). Politicas educativas en Latinoamérica: la vinculación entre los investigadores académicos y tomadores de decisiones en educación. Un análisis desde la teoría de los campos. Universitas Humanística, 83, 57-82. http:/ / dx.doi.org/10.11144/Javeriana.uh83.pelv 